\title{
The burden of excessive saturated fatty acid intake attributed to ultra-processed food consumption: a study conducted with nationally representative cross-sectional studies from eight countries
}

\author{
Eurídice Martínez Steele ${ }^{1,2}$, Carolina Batis ${ }^{3}$ (D) , Gustavo Cediel $^{2,4}$, Maria Laura da Costa Louzada ${ }^{1,2}$, \\ Neha Khandpur ${ }^{1,2,5}$, Priscila Machado ${ }^{2,6}$ D , Jean-Claude Moubarac ${ }^{7}$, Fernanda Rauber ${ }^{1,2,8}$, \\ Marcela Reyes Jedlicki ${ }^{9}$, Renata Bertazzi Levy ${ }^{2,8}$ and Carlos A. Monteiro ${ }^{1,2} *$ \\ ${ }^{1}$ Department of Nutrition, School of Public Health, University of São Paulo, São Paulo, Brazil \\ ${ }^{2}$ Center for Epidemiological Studies in Health and Nutrition, University of São Paulo, São Paulo, Brazil \\ ${ }^{3}$ CONACYT - Center for Nutrition and Health Research, National Institute of Public Health, Mexico City, Mexico \\ ${ }^{4}$ School of Nutrition and Dietetics, University of Antioquia, Medellin, Colombia \\ ${ }^{5}$ Department of Nutrition, Harvard T.H. Chan School of Public Health, Boston, USA \\ ${ }^{6}$ Institute for Physical Activity and Nutrition (IPAN), School of Exercise and Nutrition Sciences, Deakin University, Geelong 3220, Australia \\ ${ }^{7}$ Département de Nutrition, Université de Montréal, Montréal, Canada \\ ${ }^{8}$ Department of Preventive Medicine, School of Medicine, University of São Paulo, São Paulo, Brazil \\ ${ }^{9}$ CLAPEC, Unidad de Nutrición Pública, INTA, Universidad de Chile, Santiago, Chile
}

(Received 15 January 2021 - Final revision received 23 April 2021 - Accepted 30 April 2021)

Journal of Nutritional Science (2021), vol. 10, e43, page 1 of 9

doi:10.1017/jns.2021.30

Abstract

Cross-sectional nutritional survey data collected in eight countries were used to estimate saturated fatty acid intakes. Our objective was to estimate the proportion of excessive saturated fatty acid intakes ( $>10 \%$ of total energy intake) that could be avoided if ultra-processed food consumption was reduced to levels observed in the first quintile of each country. Secondary analysis was performed of $24 \mathrm{~h}$ dietary recall or food diary/record data collected by the most recently available nationally representative cross-sectional surveys carried out in Brazil (2008-9), Chile (2010), Colombia (2005), Mexico (2012), Australia (2011-12), the UK (2008-16), Canada (2015) and the US (2015-16). Population attributable fractions estimated the impact of reducing ultraprocessed food consumption on excessive saturated fatty acid intakes (above $10 \%$ of total energy intake) in each country. Significant relative reductions in the percentage of excessive saturated fatty acid intakes would be observed in all countries if ultra-processed food consumption was reduced to levels observed in the first quintile's consumption. The reductions in excessive intakes ranged from $10 \cdot 0 \%$ (95 \% CI 6.2-13.6 \%) in Canada to $35 \cdot 0 \%(95 \% \mathrm{CI}$ $28 \cdot 7-48.0 \%$ in Mexico. In all eight studied countries, all presenting more than $30 \%$ of intakes with excessive saturated fatty acids, lowering the dietary contribution of ultra-processed foods to attainable, context-specific levels was shown to be a potentially effective way to reduce the percentage of intakes with excessive saturated fatty acids, which may play an important role in the prevention of non-communicable diseases, particularly cardiovascular diseases.

Key words: Diet: Population attributable fraction: Saturated fatty acids: Ultra-processed foods

Abbreviations: CI: confidence interval; CVD: cardiovascular disease; ES: estimated size; NCD: non-communicable disease; PAF: population attributable fraction; PR: prevalence ratio; UPF: ultra-processed food

* Corresponding author: Carlos A. Monteiro, email carlosam@usp.br

(C) The Author(s), 2021. Published by Cambridge University Press on behalf of The Nutrition Society. This is an Open Access article, distributed under the terms of the Creative Commons Attribution licence (http://creativecommons.org/licenses/by/4.0/), which permits unrestricted re-use, distribution, and reproduction in any medium, provided the original work is properly cited. 


\section{Introduction}

Ultra-processed foods (UPFs), according to the NOVA classification, are industrial formulations of processed food substances (oils, fats, sugars, starch, protein isolates) that contain little or no whole food and typically include flavourings, colourings, emulsifiers and other cosmetic additives ${ }^{(1)}$. Processes and ingredients used for the manufacture of UPFs are designed to create low-cost, highly profitable products, which are extremely palatable, convenient to use (ready-to-eat and long-lasting) and are liable to displace all other NOVA food groups (unprocessed, minimally processed and processed) and the culinary preparations made with these foods ${ }^{(1)}$.

Large cohort studies carried out in different countries have shown that increased dietary share of UPFs results in a higher risk of overweight/obesity, hypertension, coronary and cerebrovascular diseases, diabetes, overall and breast cancer, and all-cause mortality ${ }^{(2-4)}$. The positive association of diets high in UPFs with excessive energy intake and weight gain was confirmed in a randomized clinical trial ${ }^{(5)}$.

Although multiple mechanisms for the association between UPF intake and non-communicable diseases (NCDs) have been envisaged ${ }^{(1)}$ including the lower satiety potential and higher glycemic index of these foods ${ }^{(1)}$ and the presence of additives such as artificial sweeteners and emulsifiers $^{(2)}$, and of compounds that are neoformed during processing or released from packaging ${ }^{(2)}$, the most obvious mechanism is their effect on the nutrient profile of the overall diet.

Indeed, nationally representative studies conducted in $\mathrm{Brazil}^{\left({ }^{(}\right)}$, Chile $^{(7)}$, Colombia ${ }^{(8)}$, Mexico ${ }^{(9)}$, Australia ${ }^{(10)}$, the $\mathrm{UK}^{(11)}$, Canada ${ }^{(12)}$ and the US ${ }^{(13)}$ have shown that increased UPF intake was associated with non-recommended intakes of most nutrients critical to NCDs, including saturated fatty acids ${ }^{(14)}$.

Although recent evidence suggests that the health effects of saturated fatty acids may vary depending on the type of fatty acid and on the specific food source ${ }^{(15)}$, a recent dietary recommendation from the World Health Organization confirmed the need of limiting saturated fatty acid intake to less than $10 \%$ of total energy intake ${ }^{(16)}$. This recommendation was based on systematic review and meta-analyses of results from all randomized controlled trials and cohort studies that have examined the association between saturated fatty acid intake and incidence of and mortality by cardiovascular disease $(\mathrm{CVD})^{(16)}$. Also based on systematic review and meta-analyses, the American Heart Association is still more restrictive and recommends limiting saturated fatty acid intake to 5-6\% of total energy intake ${ }^{(17)}$. The most recent national dietary guidelines from each of eight countries included in the present study also recommend limiting saturated fatty acid intake to reduce $\mathrm{CVDs}^{(18-25)}$.

The present paper describes for the first time, the proportion of excessive saturated fatty acid intake $(>10 \%$ of total energy intake) that could be potentially avoided, if UPF consumption was reduced to levels observed among the lowest consumers (the first quintile) across eight countries.

\section{Material and methods}

\section{Data sources}

All data used by the present study come from most recently available national dietary surveys carried out in Brazil (2008$9)^{(6)}$, Chile $(2010)^{(7)}$, Colombia $(2005)^{(8)}$, Mexico $(2012)^{(9)}$, Australia $(2011-12)^{(10)}$, the UK $(2008-16)^{(11)}$, Canada $(2015)^{(12)}$ and the US (2015-16) ${ }^{(13)}$. Relevant characteristics of these surveys, including age groups studied, sample sizes, dietary assessment methods and food composition tables, are described in Table 1. Briefly, UK used 4-d food diaries; Mexico and Canada used 1-d 24-h recalls, while the remaining countries used 2-d 24-h recalls. The analytical samples were restricted to participants with 3 or $4 \mathrm{~d}$ of food diary in the UK, one dietary recall in Mexico and Canada, two dietary recalls in Brazil and up to two dietary recalls in the remaining countries.

The present study was conducted according to the guidelines laid down in the Declaration of Helsinki, and all procedures involving human subjects were approved by the Research Ethics Committee from the Faculdade de Saúde Pública at the Universidade de São Paulo (Protocol 128958, 19 October 2012); Comité de Ética de Investigación de la Escuela de Medicina de la Pontificia Universidad Católica de Chile; Comité de ética de la Universidad del Rosario (Bogotá, Colombia); INSP Research, Biosafety, and Ethics Committee in Cuernavaca, Mexico; Australian Government Department of Health and Ageing Departmental Ethnics Committee in 2011; UK relevant research ethics and governance committees; Comité d'éthique de la recherche en sciences et en santé (CERSES) of Université de Montréal (17-017-CERES-D); US NCHS Research Ethics Review Board (ERB) Approval (Continuation of Protocol \#2011-17). Written informed consent was obtained from all subjects.

\section{Data analysis}

All analyses were run separately for each country using mean of 3 or $4 \mathrm{~d}$ of food diaries in the UK, day 1 dietary recall data in Mexico and Canada, mean day 1 and day 2 dietary recall data in Brazil, and mean day 1 and day 2 dietary recall data if available and day 1 otherwise in the remaining countries. Continuous variables presented an approximately normal distribution. First, we calculated the mean percentage of energy intake from UPFs (classified using the NOVA system $)^{(1)}$, the mean percentage of energy intake from saturated fatty acids and the percentage of intakes with excessive saturated fatty acid ( $>10 \%$ of energy intake, according to WHO recommendation $)^{(14,16)}$. Then, we used linear regression models to estimate the association between the dietary energy contribution of UPFs (categorised into quintiles) and the dietary content of saturated fatty acids (expressed as percentage of total energy). Poisson regression models with robust variance were used to assess whether the percentage of intakes with excessive saturated fatty acid increased across quintiles of the dietary energy contribution of UPFs. 


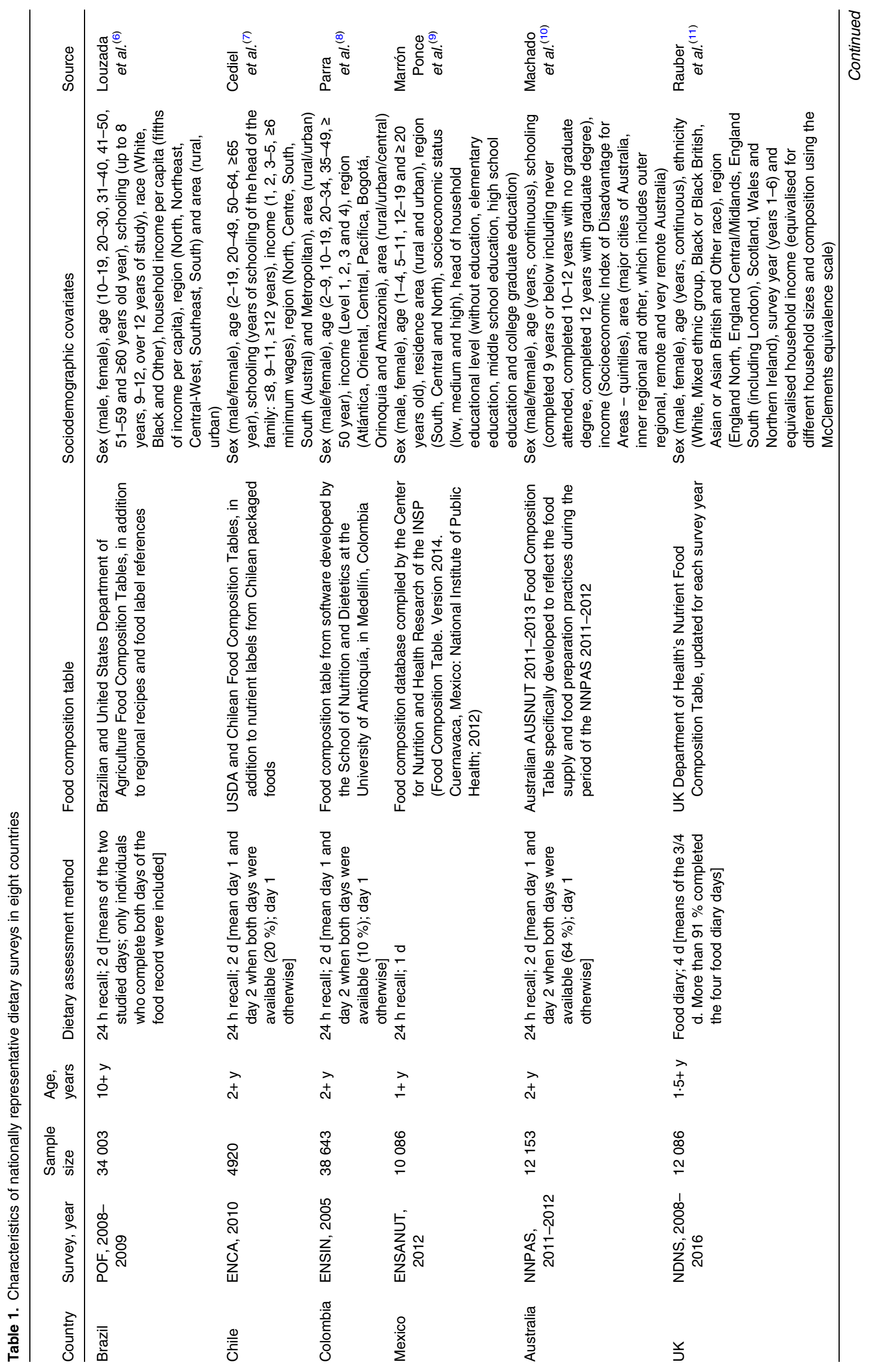




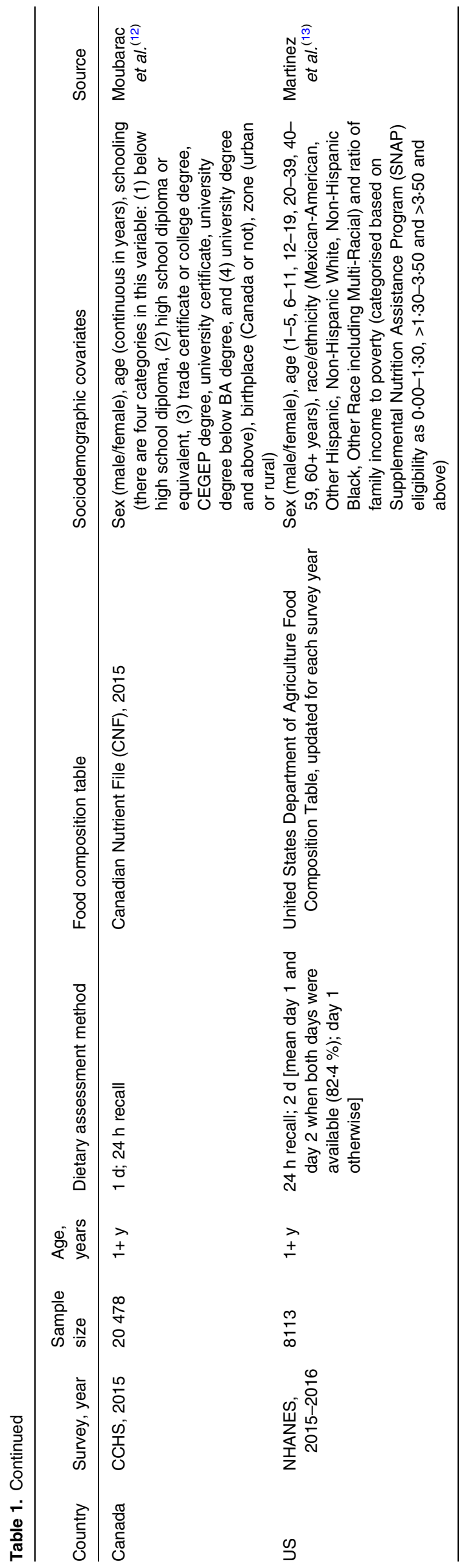

To evaluate dose-response associations, tests of linear trend were performed by treating quintiles of UPF consumption as an ordinal (1-5) variable.

Finally, we estimated, again for each country, the UPF population attributable fraction (PAF) defined as the proportion of intakes with excessive saturated fatty acid intake that would be potentially avoided if UPF consumption (the exposure) was reduced to attainable values. We assumed attainable values to be those estimated in the first quintile of UPF consumption for each country.

We calculated $\mathrm{PAF}^{(26,27)}$ through the following equation:

$$
\mathrm{PAF}=\frac{P_{\text {population }}-P_{\text {nonexposed }}}{P_{\text {population }}}
$$

where $P_{\text {population }}$ is the percentage of intakes with excessive saturated fatty acid in the total population and $P_{\text {nonexposed }}$ is the percentage of intakes with excessive saturated fatty acid in the first quintile of UPF consumption. All models were adjusted for the sociodemographic covariates displayed in Table 1. Statistical hypotheses were tested using a two-tailed $P \leq 0.05$ level of significance. All analyses were conducted with Stata statistical software package version 14 .

\section{Results}

Table 2 describes overall energy contributions of UPF and saturated fatty acid intakes across the eight studied countries. The mean energy contribution of UPFs varied from $15.9 \%$ ( $95 \%$ CI $15 \cdot 3,16.5)$ in Colombia to $56.7 \%$ (95\% CI 56.2, $57 \cdot 1)$ in the UK. Colombia showed the lowest mean dietary intake of saturated fatty acids $(8 \cdot 6 \%(95 \%$ CI $8 \cdot 4,8 \cdot 8)$ of total energy intake) and the lowest percentage of intakes with excessive saturated fatty acids $(31.40 \% \quad(95 \%$ CI $31 \cdot 38,31.42)$ ) while the highest estimates for these two parameters were observed in the UK $(12 \cdot 1 \%$ (95\% CI 12.0, 12.2) and $74.0 \%(95 \%$ CI $72 \cdot 8,75 \cdot 2)$, respectively).

As depicted in Fig. 1, the adjusted mean dietary intake of saturated fatty acids increased significantly across quintiles of the dietary contribution of UPFs in all countries ( $P$ for linear trend $<0 \cdot 05)$. Supplementary Table S1 of Supplementary material describes the mean and range of the dietary contribution of UPFs per quintile.

Table 3 shows the adjusted percentage of excessive saturated fatty acid intakes according to quintiles of the dietary contribution of UPFs. In all eight countries, the percentage of intakes with excessive saturated fatty acids increased significantly across the quintiles ( $P$ for linear trend $<0 \cdot 001$ ). The adjusted ratio of the percentage of intakes with excessive saturated fatty acid between extreme UPF quintiles ranged from $1.14(95 \%$ CI $1.07,1.22)$ in Canada up to $2.05(95 \%$ CI $1 \cdot 65,2.54)$ in Chile (Fig. 2). The values in the first $v$. the last quintile almost doubled in Brazil, Mexico and Chile.

If the dietary contribution of UPFs was reduced to levels observed in the first quintile of each country, statistically significant reductions in the percentage of excessive saturated fatty acid intakes would be observed in all eight countries. The reductions would vary from $10 \cdot 0 \%$ (95\% CI 6.2, 13.6) 
Table 2. Contribution of UPFs and saturated fatty acids to total energy intake (kcal/d) and prevalence of excessive saturated fatty acid intakes in eight countries

\begin{tabular}{lcccc}
\hline Country & $\begin{array}{c}\text { Total energy intake } \\
(\mathrm{kcal} / \mathrm{d})\end{array}$ & $\begin{array}{c}\text { Percentage of total energy } \\
\text { intake from UPFs } \\
\text { mean }(95 \% \mathrm{Cl})\end{array}$ & $\begin{array}{c}\text { Percentage of total energy intake from } \\
\text { saturated fatty acids } \\
\text { mean }(95 \% \mathrm{Cl})\end{array}$ & $\begin{array}{c}\text { Prevalence of intakes with excessive } \\
\text { saturated fatty acids } \\
\%(95 \% \mathrm{Cl})\end{array}$ \\
\hline Colombia & $1835 \cdot 1(1811 \cdot 6,1858 \cdot 6)$ & $15 \cdot 9(15 \cdot 3,16 \cdot 5)$ & $8 \cdot 6(8 \cdot 4,8 \cdot 8)$ & $31 \cdot 40(31 \cdot 38,31 \cdot 42)$ \\
Brazil & $1952 \cdot 9(1932 \cdot 5,1973 \cdot 3)$ & $18 \cdot 9(18 \cdot 2,19 \cdot 2)$ & $9 \cdot 7(9 \cdot 6,9 \cdot 8)$ & $40 \cdot 8(39 \cdot 7,41 \cdot 9)$ \\
Chile & $1804 \cdot 7(1760 \cdot 6,1848 \cdot 8)$ & $28 \cdot 6(27 \cdot 7,29 \cdot 6)$ & $8 \cdot 8(8 \cdot 7,9 \cdot 0)$ & $32 \cdot 4(30 \cdot 2,34 \cdot 7)$ \\
Mexico & $1923 \cdot 4(1886 \cdot 2,1960 \cdot 6)$ & $30 \cdot 0(29 \cdot 2,30 \cdot 8)$ & $11 \cdot 2(11 \cdot 0,11 \cdot 4)$ & $58 \cdot 4(56 \cdot 5,60 \cdot 3)$ \\
Australia & $1926 \cdot 5(1908 \cdot 5,1944 \cdot 5)$ & $41 \cdot 6(41 \cdot 1,42 \cdot 1)$ & $12 \cdot 0(11 \cdot 8,12 \cdot 1)$ & $67 \cdot 1(66 \cdot 0,68 \cdot 2)$ \\
Canada & $1816 \cdot 1(1804 \cdot 7,1827 \cdot 5)$ & $51 \cdot 0(50 \cdot 6,51 \cdot 4)$ & $11 \cdot 0(10 \cdot 9,11 \cdot 1)$ & $56 \cdot 80(56 \cdot 79,56 \cdot 81)$ \\
US & $1995 \cdot 1(1956 \cdot 9,2033 \cdot 4)$ & $56 \cdot 2(55 \cdot 0,57 \cdot 3)$ & $11 \cdot 5(11 \cdot 3,11 \cdot 7)$ & $66 \cdot 9(64 \cdot 2,69 \cdot 6)$ \\
UK & $1746 \cdot 7(1732 \cdot 91760 \cdot 6)$ & $56 \cdot 7(56 \cdot 2,57 \cdot 1)$ & $12 \cdot 1(12 \cdot 0,12 \cdot 2)$ & $74 \cdot 0(72 \cdot 8,75 \cdot 2)$ \\
\hline
\end{tabular}

in Canada all the way up to $35 \cdot 0 \%(95 \%$ CI $28 \cdot 7,40 \cdot 8)$ in Mexico (Fig. 3).

\section{Discussion}

In the present study across eight middle- and high-income countries, we observed that the rise in UPF consumption was systematically associated with an increase in both the mean saturated fatty acid intakes and in the percentage of excessive intakes. The percentage of excessive saturated fatty acid intakes would be significantly reduced in all countries (between $10 \%$ in Canada and $35 \%$ in Mexico), if UPF consumption was decreased to context-specific attainable levels such as those estimated for the lowest quintile of UPF consumption in each country.

Despite the fact that not all UPF items are a source of saturated fatty acids and some of them are even exempt of fat, soft drinks being an example, our findings show that, irrespective of the country, dietary patterns characterised by relatively higher overall contribution of UPFs tend to have a higher content of saturated fatty acids. One likely explanation is the frequent use of saturated fatty acid rich crude palm oil $(51 \%$ content of saturated fatty acids) and palm kernel oil (84\% content of saturated fatty acids) ${ }^{(28)}$ in the manufacture of several UPFs such as baked goods, candies, cakes, cheese analogues, chips, chocolate, confectionary fats, cookies, crackers, doughnuts, frozen meals (pancakes, pies, pizza, potatoes), ice cream, instant noodles, margarines, shortenings and other butter substitutes, microwave popcorn, non-dairy creamers, salad dressings, snacks, soups, among others ${ }^{(29,30)}$. The preference for these oils and their fractions and blends lies in their fragrance and neutral taste and in their capacity to confer the texture obtained with the use of butter or lard (without the need of using hydrogenation, thus reducing trans-fat content) as well

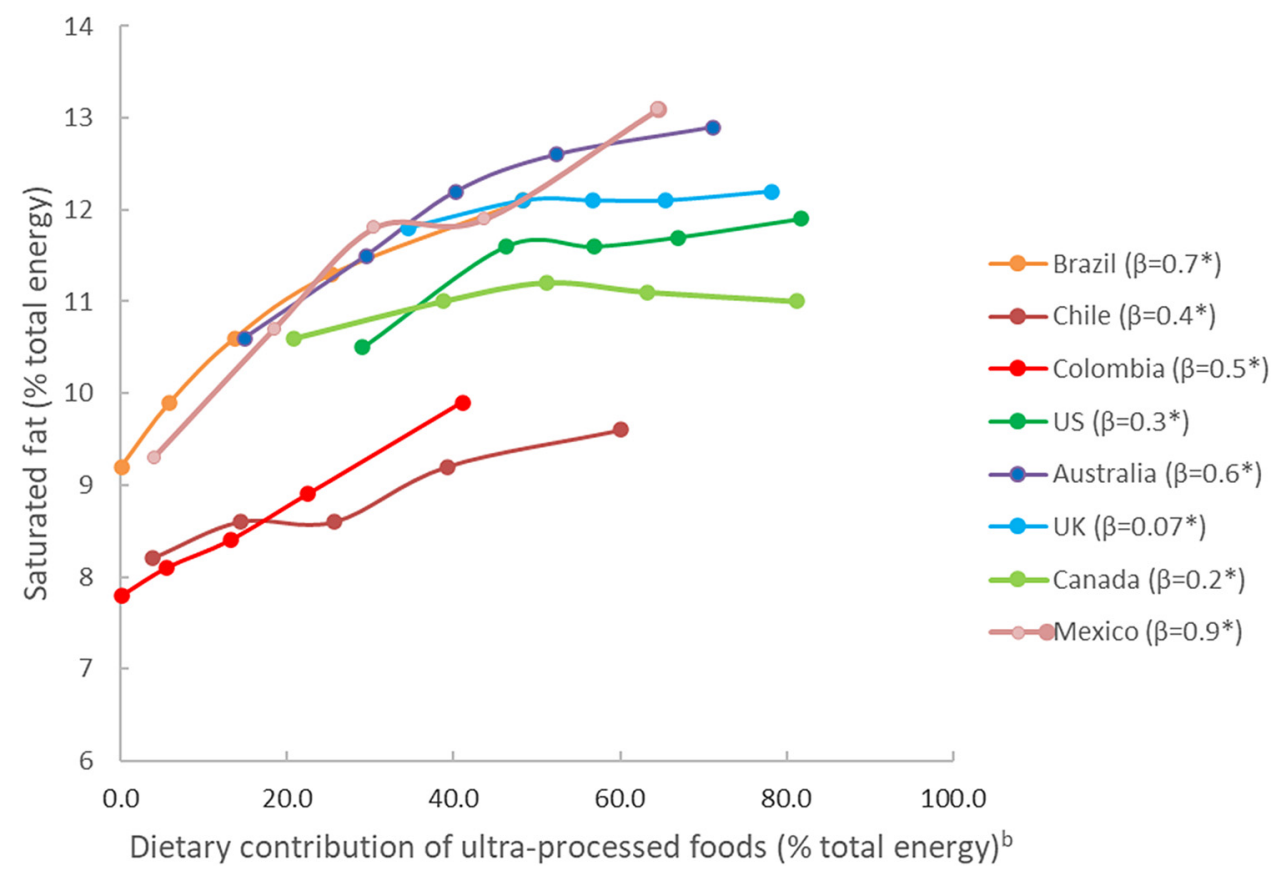

Fig. 1. Adjusted ${ }^{\mathrm{a}}$ mean dietary content of saturated fatty acids across quintiles of the dietary contribution of UPFs in eight countries. ${ }^{\mathrm{a}}$ Adjusted according to covariates listed in Table $1 .{ }^{b}$ Knots corresponding to country-specific quintiles of the dietary contribution of UPFs. * Statistically significant coefficient for linear trend $(P<$ 0.05). 
Table 3. Adjusted ${ }^{a}$ percentage of intakes with excessive saturated fatty acids $(>10 \%)$ across quintiles of the dietary contribution of UPFs in eight countries

\begin{tabular}{|c|c|c|c|c|c|c|}
\hline \multirow[b]{2}{*}{ Country } & \multicolumn{5}{|c|}{ Quintiles of the dietary contribution of UPFs ${ }^{a}$} & \\
\hline & Q1 & Q2 & Q3 & Q4 & Q5 & \\
\hline Colombia & $24.9(22.9 ; 26.9)$ & $27 \cdot 1(25 \cdot 1 ; 29 \cdot 1)$ & $29.4(27.4 ; 31.4)$ & $33.8(31.8 ; 35 \cdot 8)$ & $40 \cdot 2(38.2 ; 42 \cdot 2)$ & \\
\hline Brazil & $31.6(29.6 ; 33.6)$ & $32.6(30.7 ; 34.5)$ & $36.1(34.1 ; 38.1)$ & $44 \cdot 3(42 \cdot 1 ; 46 \cdot 5)$ & $57.4(55.0 ; 59.7)$ & * \\
\hline Chile & $22.5(18 \cdot 7 ; 26 \cdot 3)$ & $26.8(21.7 ; 31.9)$ & $27 \cdot 6(23 \cdot 2 ; 32 \cdot 0)$ & $37.9(33.0 ; 42.8)$ & $46 \cdot 2(40 \cdot 3 ; 52 \cdot 0)$ & * \\
\hline Mexico & $38.6(34.5 ; 42.7)$ & $51.8(48.2 ; 55.4)$ & $64.2(60.6 ; 67.7)$ & $67.7(63.9 ; 71.6)$ & $72.5(69.0 ; 75.9)$ & * \\
\hline Canada & $51.40(51.38 ; 51.42)$ & $56.60(56.58 ; 56.62)$ & $58.90(58 \cdot 88 ; 58.92)$ & $58 \cdot 20(58 \cdot 18 ; 58 \cdot 22)$ & $58.60(58.58 ; 58.62)$ & * \\
\hline Australia & $50.4(47 \cdot 6 ; 53 \cdot 1)$ & $62.9(60 \cdot 2 ; 65.6)$ & $69 \cdot 2(66 \cdot 7 ; 71 \cdot 6)$ & $75.1(72.9 ; 77.4)$ & $77 \cdot 3(75 \cdot 1 ; 79.6)$ & * \\
\hline US & $53 \cdot 8(47.5 ; 60 \cdot 1)$ & $67 \cdot 1(63 \cdot 0 ; 71 \cdot 1)$ & $69.5(66 \cdot 6 ; 72 \cdot 5)$ & $71 \cdot 9(69 \cdot 1 ; 74 \cdot 6)$ & $71.9(67 \cdot 6 ; 76 \cdot 2)$ & * \\
\hline UK & $65.5(62 \cdot 3 ; 68.7)$ & $73 \cdot 3(70 \cdot 5 ; 76 \cdot 1)$ & 76.1 (73.5; 78.8) & $75 \cdot 7(73 \cdot 1 ; 78 \cdot 3)$ & $80 \cdot 1(77.7 ; 82.4)$ & * \\
\hline
\end{tabular}

${ }^{a}$ Adjusted according to covariates listed in Table $1.95 \% \mathrm{Cl}$ in parenthesis.

${ }^{*}$ Statistically significant linear trend $(P \leq 0 \cdot 05)$.

as to increase the thermal and oxidative stability of the fat, and thus the shelf life of the final product ${ }^{(28,29)}$. The high productivity of palm oil, which is able to produce up to ten times more oil per hectare of plantation than other leading oilseed crops $^{(31)}$, may be an additional economic force driving its use in UPFs. In fact, in 2012, crude palm oil and palm kernel oil (both extracted from palm fruit) overtook soybean oil as the most important vegetable oil in the world, accounting for $32 \%$ of global fats and oils production, $90 \%$ of which being used by the edible food industry ${ }^{(29,31)}$.

The relationship found between UPF consumption and excessive saturated fatty acid intake indicates that these intakes may be one of the mechanisms for the prospective association between UPF consumption and both cardiovascular diseases ${ }^{(2,32)}$ and several major risk factors for these diseases, including overweight and obesity, hypertension, dyslipidaemia and diabetes ${ }^{(2,3,33)}$.

Taxes, front-of-pack labels and dietary guidelines are potential vehicles that can be used to discourage the consumption of
UPFs. However, while reducing UPF consumption to levels observed in the first quintile would help, this is not enough to eliminate excessive saturated fatty acid intake. Australia, UK, Canada and US would still have over $50 \%$ percentage of excessive intakes, Brazil and Mexico over $30 \%$, and Chile and Colombia over $20 \%$. Especially in the UK and US but also in Australia and Canada, with already significant UPF consumption in the first quintile, countries would need to set goals to reduce UPFs well below levels observed in the first quintile. In Brazil, Mexico, Chile and Colombia, in which mean UPF contribution is already below $5 \%$ in the first quintile, countries would need to extend the reduction to non-ultra-processed sources of saturated fatty acids such as meat, butter and cheese.

On the other hand, in all eight countries evaluated in the present study, UPF consumption was shown to be inversely related to protein and fibre intakes and directly related to free sugar intake ${ }^{(6-13)}$ suggesting that UPFs tend to replace healthier

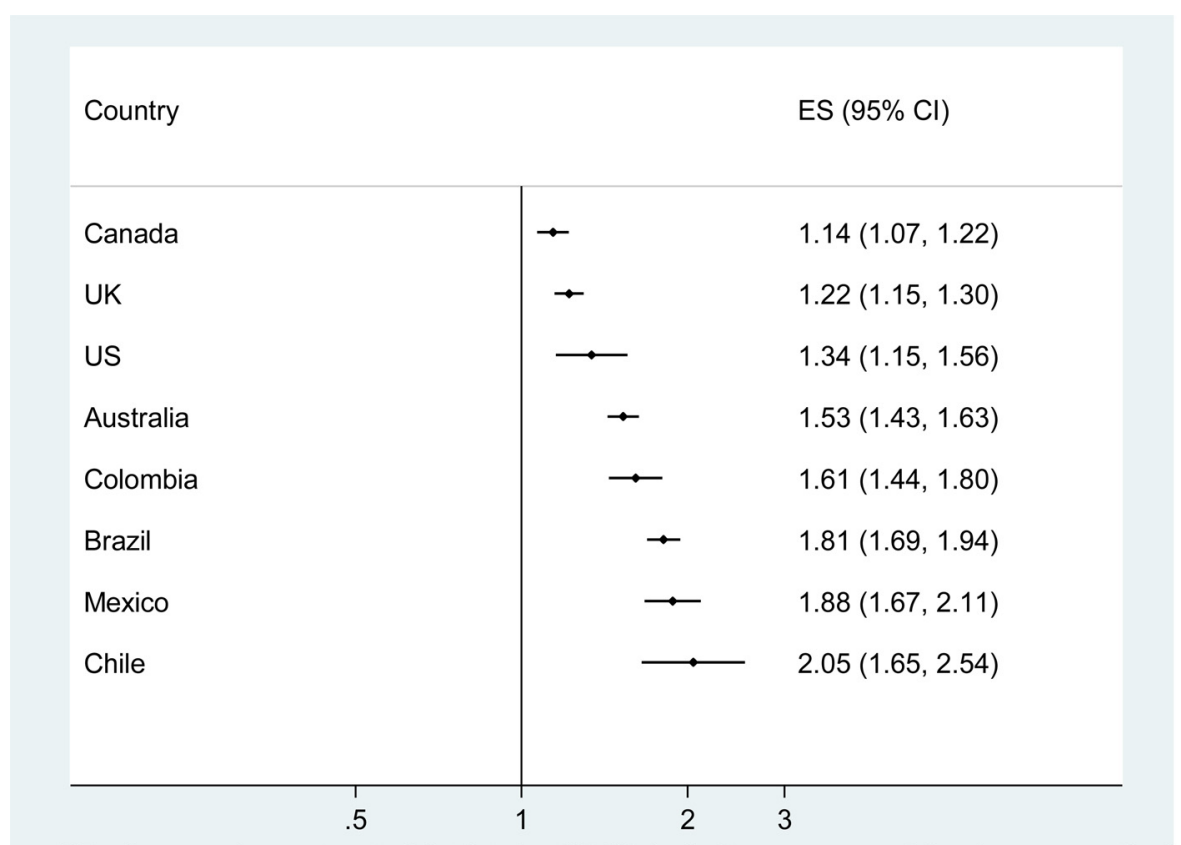

PR of excessive saturated fat intake (Q5/Q1 of ultra-processed food consumption)

Fig. 2. Adjusted ${ }^{\mathrm{a}} \mathrm{PR}$ of excessive saturated fatty acid intake (>10\%) between extreme quintiles of the dietary contribution of UPFs in eight countries. ${ }^{\mathrm{a}}$ Adjusted according to covariates listed in Table 1. 


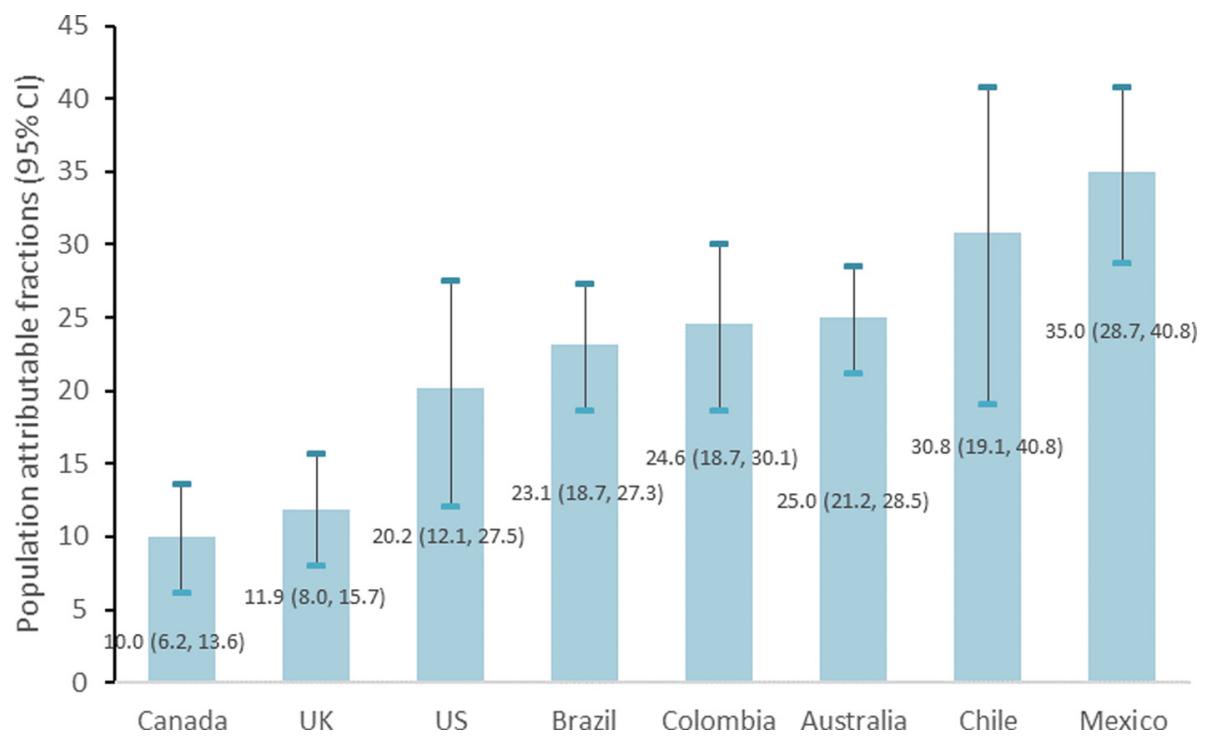

Fig. 3. Impact of UPF consumption on the adjusted ${ }^{a}$ prevalence of excessive saturated fatty acid intake in eight countries. ${ }^{\mathrm{a}}$ Adjusted according to covariates listed in Table 1.

sources of food and that reducing its consumption would probably entail additional cardiovascular and other health benefits.

Strengths of the present study include the use of large, nationally representative samples of the population of eight middle- and high-income countries, maximising external validity of results. The use of individual consumption data rather than market disappearance or household purchasing data, which may under- or overestimate amounts consumed, is an additional strength of the present study as well as the fact that the NOVA classification was applied similarly across all countries after disaggregating handmade recipes into underlying ingredients, maximising the comparability across settings. The use of context-specific scenarios across the countries for estimating the reduction in the percentage of intakes with excessive fatty acids is also a strength of the present study.

Though the most recent surveys were used for each country, the years of data collection were less recent in some countries possibly not reflecting actual consumption and also limiting comparisons across countries. Despite existing evidence of the increasing trends in UPF consumption in many countries ${ }^{(34)}$, how this should affect the population attributable fractions is less clear because it depends much more on the difference in risk between the first and all other quintiles than on the absolute contribution of UPFs to the diet. Though sample sizes varied from country to country ranging from 4920 in Chile to 38643 in Colombia, all provided statistically significant and relatively precise PAFs (see Fig. 3). Random and systematic error may bias self-reported dietary intake data collected through $24-\mathrm{h}$ recall/food diaries, especially regarding absolute energy intakes, even though the applied standardised methods should contribute to minimising it, and also the fact that we always used relative estimates. Though social desirability bias may lead to an underestimation of the dietary contribution of UPFs and of saturated fatty acids, this would most likely not affect the association between the two variables or it would bias the association towards the null. Despite these limitations inherent to self-reported diet, 24-h recalls/food diaries are considered the least biased method and the best for describing dietary intake at the population level ${ }^{(35)}$. In most countries, the assessment of dietary intakes was exclusively or mostly based on $1 \mathrm{~d}$ recalls potentially overestimating the frequency of individuals with very low or very high long-term saturated fatty acid intakes ${ }^{(36)}$. Though most countries included participants aged 1 or 2 years onwards, in Brazil only participants aged 10 years and older were included. If as shown in other countries such as the US ${ }^{(37)}$, children consume more UPFs than adults, UPF consumption, saturated fatty acids and thus, PAF, of the overall population may be underestimated in Brazil.

In addition, although all studies collected some information indicative of the type of processing to which foods were submitted before consumption or culinary preparation (i.e. place of meals and product brands), this information was not available for all food items, which could lead to modest over- or underestimation of UPF consumption. An additional source of error may be the use of combined foreign and national food composition tables in some countries (i.e. Brazil and Chile). Also, reformulation could have decreased saturated fatty acid content of UPFs since data collection, though the fact that associations persisted in studies with more recent data suggests that this is unlikely the case. Finally, PAFs need to be interpreted with caution especially because these are applied to population estimates and do not account for individual-level behaviour changes including food substitutions, replacements and abandonment effects associated with the reduction in UPF consumption.

In summary, the present study, carried out in nationally representative samples of the population of eight middle- and high-income countries, indicates that lowering the dietary contribution of UPFs to attainable, context-specific levels is a potentially effective way to reduce saturated fatty acid intake 
and the percentage of intakes with excessive content, which may play an important role in the prevention of NCDs, particularly cardiovascular diseases.

\section{Supplementary material}

The supplementary material for this article can be found at https://doi.org/10.1017/jns.2021.30.

\section{Acknowledgments}

This work was supported by the Fundação de Amparo à Pesquisa do Estado de São Paulo (FAPESP) [with grant numbers 2015/14900-9; 2016/14302-7; 2018/17972-9].

E. M. S., R. B. L. and C. A. M. formulated the research question and designed the study; E. M. S., C. B., G. C., M. L. C. L., N. K., P. M., J.-C. M. and F. R. analysed the data; E. M. S., C. B., G. C. M. L. C. L., N. K., P. M., J.-C. M., F. R., M. R. J., R. B. L. and C. A. M. wrote the article.

E. M. S., F. R. are beneficiaries of Postdoctoral Fellowship. Fundação de Amparo à Pesquisa do Estado de São Paulo (FAPESP) had no role in the design, analysis or writing of this article.

There are no conflicts of interest.

\section{References}

1. Monteiro CA, Cannon G, Levy RB, et al. (2019) Ultra-processed foods: What they are and how to identify them. Public Health Nutr 22, 936-941. doi:10.1017/S1368980018003762.

2. Srour B, Fezeu LK, Kesse-Guyot E, et al. (2019) Ultra-processed food intake and risk of cardiovascular disease: Prospective cohort study (NutriNet-Santé). Br Med J 365, 11451. doi:10.1136/bmj. 11451.

3. Srour B, Fezeu LK, Kesse-Guyot E, et al. (2019) Ultraprocessed food consumption and risk of type 2 diabetes among participants of the NutriNet-Santé prospective cohort. JAMA Intern Med. doi:10.1001/jamainternmed.2019.5942.

4. Schnabel L, Kesse-Guyot E, Allès B, et al. (2019) Association between ultraprocessed food consumption and risk of mortality among middle-aged adults in France. JAMA Intern Med 179, 490498. doi:10.1001/jamainternmed.2018.7289.

5. Hall KD, Ayuketah A, Brychta R, et al. (2019) Ultra-processed diets cause excess calorie intake and weight gain: An inpatient randomized controlled trial of ad libitum food intake. Cell Metab 30, 67-77.e3. doi:10.1016/j.cmet.2019.05.008. Epublication 6 May 2019.

6. Costa Louzada ML, Martins AP, Canella DS, et al. (2015) Ultra-processed foods and the nutritional dietary profile in Brazil. Rev Saude Publica 49, 38. doi:10.1590/S0034-8910. 2015049006132.

7. Cediel G, Reyes M, Corvalán C, et al. (2020) Ultra-processed foods drive to unhealthy diets: Evidence from Chile. Public Health Nutr, 1-10. [Epub ahead of print]. doi:10.1017/S1368980019004737. PMID: 32338229.

8. Parra DC, da Costa-Louzada ML, Moubarac JC, et al. (2019) Association between ultra-processed food consumption and the nutrient profile of the Colombian diet in 2005. Salud Publica Mex 61, 147-154. doi:10.21149/9038.

9. Marrón-Ponce JA, Flores M, Cediel G, et al. (2019) Associations between consumption of ultra-processed foods and intake of nutrients related to chronic non-communicable diseases in Mexico. J Acad Nutr Diet 119, 1852-1865. doi:10.1016/j. jand.2019.04.020.
10. Machado PP, Martinez Steele E, Levy R, et al. (2019) Ultra-processed foods and recommended intake levels of nutrients linked to non-communicable diseases in Australia: Evidence from a nationally representative cross-sectional study. BMJ Open 9, e029544. doi:10.1136/bmjopen-2019-029544.

11. Rauber F, da Costa Louzada ML, Steele EM, et al. (2018) Ultra-processed food consumption and chronic non-communicable diseases-related dietary nutrient profile in the UK (2008-2014). Nutrients 10. doi:10.3390/nu10050587.

12. Moubarac JC, Batal M, Louzada ML, et al. (2017) Consumption of ultraprocessed foods predicts diet quality in Canada. Appetite 108, 512-520. doi:10.1016/j.appet.2016.11.006. Epublication 4 November 2016.

13. Martínez Steele E, Popkin BM, Swinburn B, et al. (2017) The share of ultra-processed foods and the overall nutritional quality of diets in the US: Evidence from a nationally representative cross-sectional study. Popul Health Metr 15, 6. doi:10.1186/s12963-017-0119-3.

14. World Health Organization (2018) Healtby Diet. Fact Sheet No. 394. Geneva: World Health Organization.

15. Astrup A, Bertram HC, Bonjour JP, et al. (2019) WHO draft guidelines on dietary saturated and trans fatty acids: Time for a new approach? BMJ 366, 14137. doi:10.1136/ bmj.14137.

16. WHO (2018) https://extranet.who.int/dataform/upload/surveys/ 666752/files/Draft $\% 20$ WHO $\% 20$ SFA-TFA\%20guidelines_0405 2018\%20Public\%20Consultation(1).pdf, 27/May/2021.

17. Van Horn L, Carson JAS, Appel LJ, et al. (2016) Recommended dietary pattern to achieve adherence to the American Heart Association/ American College of Cardiology (AHA/ACC) guidelines: A scientific statement from the American Heart Association. Circulation 134, e505-e529. doi:10.1161/CIR.0000000000000462.

18. National Health and Medical Research Council (2013) Australian Dietary Guidelines. Canberra, Australia: National Health and Medical Research Council.

19. Guia Alimentar para a População Brasileira [Dietary Guidelines for the Brazilian population]. Brasília - DF (2014).

20. Public Health England (2019) Canada's Dietary Guidelines for Health Professionals and Policy Makers. Her Majesty the Queen in Right of Canada, as represented by the Minister of Health.

21. Dietary guidelines for the Chilean population. Ministry of Health, May 16th 2013.

22. Guías Alimentarias para la población Colombiana mayor de 2 años Basadas en Alimentos [Food-based dietary guidelines for the Colombian population over 2 years of age]. Noviembre (2015).

23. Guías alimentarias y de actividad física en contexto de sobrepeso y obesidad en la población mexicana [Dietary and physical activity guidelines in the context of overweight and obesity in the Mexican population]. Marzo de (2015).

24. https://www.gov.uk/government/publications/the-eatwell-guide

25. Dietary Guidelines for Americans 2015-2020, 8th ed.

26. Steenland K \& Armstrong B (2006) An overview of methods for calculating the burden of disease due to specific risk factors. Epidemiology 17, 512-519.

27. Rezende LFM \& Eluf-Neto J (2016) Population attributable fraction: Planning of diseases prevention actions in Brazil. Rev Saúde Pública 50, 30.

28. Vaclavik VA \& Christian EW (2014) Essentials of Food Science, 4th ed., US: Springer.

29. Mba OI, Dumont MJ \& Ngadi M (2015) Palm oil: Processing, characterization and utilization in the food industry - A review. Food Bioscience 10, 26-41. doi:10.1016/j.fbio.2015.01.003.

30. Mancini A, Imperlini E, Nigro E, et al. (2015) Biological and nutritional properties of palm oil and palmitic acid: Effects on health. Molecules 20, 17339-17361, published online 18 September 2015. doi:10.3390/molecules200917339.

31. Oil World (2013) Oil world annual 2013. http://www.oilworld.biz/ app.php. (released June 2013).

32. Bonaccio M, Di Castelnuovo A, Costanzo S, et al. (2020) Consumption of ultra-processed foods and beverages is associated with increased risk of cardiovascular mortality in the Moli-sani Study Cohort. Circulation 141, A49. 
33. Rauber F, Campagnolo PD, Hoffman DJ, et al. (2015) Consumption of ultra-processed food products and its effects on children's lipid profiles: A longitudinal study. Nutr Metab Cardiovasc Dis 25, 116-122. doi:10.1016/ j.numecd.2014.08.001.

34. Vandevijvere S, Jaacks LM, Monteiro CA, et al. (2019) Global trends in ultraprocessed food and drink product sales and their association with adult body mass index trajectories. Obes Rev 20, 10-19. doi:10.1111/obr.12860. Epublication 17 May 2019.

35. Prentice RL, Mossavar-Rahmani Y, Huang Y, et al. (2011) Evaluation and comparison of food records, recalls, and frequencies for energy and protein assessment by using recovery biomarkers. Am. J. Epidemiol 174, 591-603.

36. Herrick KA, Rossen LM, Parsons R, et al. (2018) Estimating usual dietary intake from national health and nutrition examination survey data using the National Cancer Institute method. Vital Health Stat 2 (178), National Center for Health Statistics.

37. Baraldi LG, Steele EM, Canella DS \& Monteiro CA (2018) Consumption of ultra-processed foods and associated sociodemographic factors in the USA between 2007 and 2012: Evidence from a nationally representative cross-sectional study. BMJ Open 8, e020574. doi:10.1136/bmjopen-2017-020574. 